\title{
Laboratory Test Results Directionality
}

National Cancer Institute

\section{Source}

National Cancer Institute. Laboratory Test Results Directionality. NCI Thesaurus. Code C119845.

A qualifier for the direction on the body the laboratory test is performed. 